\title{
Fundamental Property Shifts in MIMO V-Interconnected Systems for Control and Diagnostics
}

Key Words: MIMO systems; interconnections; system property changes; interaction coefficient; diagnostic symptoms.

Abstract. Theoretically inherent properties in the class of MIMO $V$-interconnected systems incline options for arising arbitrary shifts in their fundamental properties, thus demonstrating full-scale plant behaviors when running in a large process range. This curious effect leads to far more serious functional changes in the process properties from mere plant parameter variations. The phenomenon is commented occasionally by some authors in somewhat empirical forms, but nevertheless, it is not yet comprehensively examined in the light of the aggravating subsequences to the control efforts and the task of efficient process diagnostics to that class of plants.

\section{Problem Statement}

Process properties of MIMO control systems have been often treated in a conventional classic way with regard to their parameter changes due to operational variations up to now. In cases of functioning at larger working areas, however, unusual phenomena as abrupt behavior jumps or even sudden falls to instability are rarely observed and reported with having no sound justifications. A little bit from the numerous industry examples concerning these phenomena include selected hydraulic processes, operation of vessels with water-vapor content, special industrial separation units, etc., usually having technology feedback(s). Surprisingly, the available control systems don't guarantee reliable operational results of the particular plants during regular and occasional process runs. Implementation of diagnostic support gives less or more positive effects, but evidently dealing on the "following-the-disaster" principle. The aim of this note concerns clearing the causes for the fundamental property jumps in the behavior of MIMO V-interconnected systems from a theoretically justified point of view to give distinct basis for more efficient control policies and adopting new theoretical diagnostic symptoms in technology industry.

\section{Current State of the Art}

Early investigations on MIMO interconnected systems are being limited only to the analysis of their structural properties. This was imposed by the predominantly analytical modelling approach, giving physically plausible results about the inner process connections. Mesarović [4] introduces the notion of canonical structures in MIMO system theo- ry, confining the observed system variety to a couple of basic interconnecting mechanisms: the one of $P$ - or those with $V$-type $[1,3,6,7,9]$. Novel investigations [11] show however, that diverse structural mixes are also feasible and they do practically exist. Later on, in [1] R. Isermann examines in a structural way the input-output properties of the simplest (2x2) interconnected systems of P- and partly those with $\mathrm{V}$-interactions, so deriving general I-O results about MIMO P-structures. Via formal reformulation of a V-canonical MIMO structure to corresponding P-type I-O representation, he also demonstrated the seeming equivalence between the P- and V-structures, but having no strict examination and system property analysis. New investigations prove [11], however, that principal distinctions between the basic MIMO structures are available. So, it is principally incorrect to formally apply uniform mathematical descriptions to both of them by neglecting their strong theoretical internal distinctions.

Johansson shows in [2] experimental results from investigating the benchmark four-tank hydraulic laboratory rig having particular circulation of flows between vessels. The developed and tested dynamic model of the hydraulic rig contains a real multivariable system zero, that reverses its sign (jump to non-minimum phase behaviour) according to some constructive parameters and process operating conditions. Indeed, the fundamental changes in plant properties are caused by its structural variations. In the reference cited, a valid physical interpretation of the phenomenon is given and the possibility of emerging other system behaviours are also suggested, due to the influence of the process cross connections.

On the other hand, distinct dynamic behaviors by structurally identical MIMO plants, consisting of two bottom-coupled hydraulic tanks (figure 1) are reported in [5] and [10]. Identical TITO interconnected models in both reports are proposed, though having similar components. In [10], the separate TF sub-models and the adjacent level outputs $h_{i}$ demonstrate intrinsic self-control. In [5], however, the corresponding level dynamics demonstrate astatic behavior. This paradox may solely be explained via the adopted operating characteristics of the effluent flows in [10], bearing overdamped decreasing dependency by the tank levels. As for the model in [5], it is evidently assumed, that the effluent tank flows stay constant and being not significantly influenced by the liquid level interactions of the tanks. 


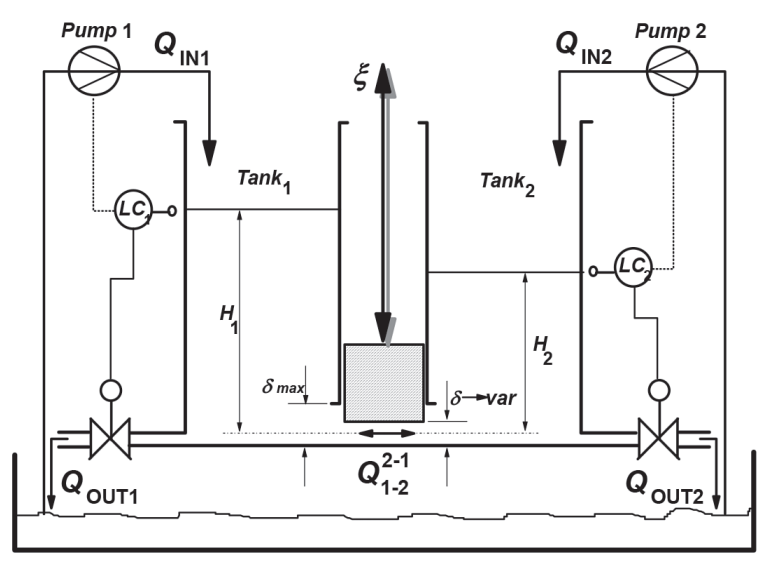

Figure 1. Principle diagram of two coupled hydraulic tanks

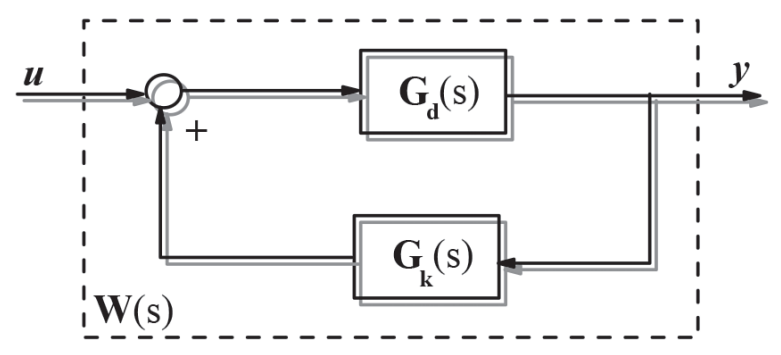

Figure 2. MIMO V-plant (7)

\section{Coupled Double-inlet Hydraulic Tank Models}

Various compound schemes for coupling of two hydraulic tanks do exist in industry [5,7,10]. In [7] a simplified version with single influent/effluent flow is considered (figure $1, Q_{\mathrm{IN} 2}=0, Q_{\mathrm{OUT1}}=0$ ) with a tank bottom connection. It refers also to MIMO V-interconnectivity [11]. The $(2 \times 2)$ model of the liquid levels $h 1, h 2$ in both vessels with changes in the input-output flows $F_{0}, F_{2}$ and bottom cross connection ( $F_{1}$ in both directions, due to vessel levels) is represented in general form by the non-linear system (1) in small quantities:

(1)

$$
\mid \begin{aligned}
& S_{1} \frac{d h_{1}}{d t}=F_{0}-F_{1}, \quad F_{1}=c_{1}\left(h_{1}-h_{2}\right) \\
& S_{2} \frac{d h_{2}}{d t}=F_{1}-F_{2}, \quad F_{2}=c_{2} h_{2}
\end{aligned}
$$

(1) The TITO plant model (1) is illustrated by the principle diagram on figure 2, containing the backward cross influences $h i-u j$ as $\mathbf{G}(\mathrm{s})$. After some assumptions, this model may be transformed into linear form (2):

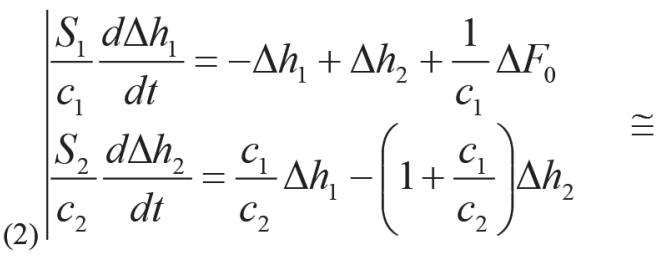

$$
\cong \mid \begin{aligned}
& a_{1} \frac{d y_{1}}{d t}+y_{1}-y_{2}=k u \\
& a_{2} \frac{d y_{2}}{d t}-a_{3} y_{1}+\left(1+a_{3}\right) y_{2}=0
\end{aligned}
$$

The same physical plant, but supplied with second influent flow $\left(F_{i}\right)$ and double effluent $G_{i}$ from both vessels owes the following complicated in comparison to (1) model description [7]:

(3)

$$
\text { (3) } \mid \begin{aligned}
& \rho S_{1} \frac{d h_{1}}{d t}=F_{1}-G_{1}-F_{12}, \quad F_{12}=S_{12} k\left(h_{1}-h_{2}\right) \\
& \rho S_{2} \frac{d h_{2}}{d t}=F_{2}-G_{2}+F_{12}, G_{i}=a \sqrt{2 g h_{i}}, F_{i} \equiv u_{i}
\end{aligned}
$$

After linearization of the non-linear functions (dependent by the levels hi) in the effluent flows $G_{i}$, it yields:

(4)

$$
\mid \begin{aligned}
& \rho S_{1} \frac{d y_{1}}{d t}=u_{1}-\frac{G_{10}}{2 h_{10}} y_{1}-k_{12}\left(y_{1}-y_{2}\right) \\
& \rho S_{2} \frac{d y_{2}}{d t}=u_{2}-\frac{G_{20}}{2 h_{20}} y_{2}+k_{12}\left(y_{1}-y_{2}\right) .
\end{aligned}
$$

According to [5], however, the model to the same tank configuration is described in a different way under definite assumptions $\left(G_{i}=\right.$ const $\left._{i}\right)$ due to the special properties of the outflows:

$$
\begin{aligned}
& \mid \begin{array}{l}
S_{1} \frac{d h_{1}}{d t}=F_{1}-G_{1}-F_{12}, \quad F_{12}=k_{12}\left(h_{1}-h_{2}\right) \\
S_{2} \frac{d h_{2}}{d t}=F_{2}-G_{2}+F_{12}, u_{i}=F_{i}-G_{i}^{C O N S T}
\end{array} \\
& \mid \begin{array}{l}
S_{1} \frac{d y_{1}}{d t}=u_{1}-k_{12}\left(y_{1}-y_{2}\right), \\
S_{2} \frac{d y_{2}}{d t}=u_{2}+k_{12}\left(y_{1}-y_{2}\right) .
\end{array}
\end{aligned}
$$

It follows, that under level invariant outflows each system output (the level change) will depend only by $u_{i}$ and will possess no self-control. By this reason, qualitative evaluation of the phenomena is relevant, that sets a non-trivial task, especially under changeable constructive characteristics and load conditions. The problem consists of both theoretical obscurity and existing fine processes, often disregarded in gross engineering considerations. So, as a consequence of the fundamental dynamic variability established, special functional tasks are to be undergone to the MIMO V-systems via a new grade of sophistication for control on large process ranges.

Incorrect structure selection to MIMO V-canonical systems

- Complex industrial systems are often modeled on a "do-it-on-parts" principle at the early identification stages, because this is the easiest way to compose input-output matrix transfer function model, instead of a sophisticated general MIMO description in form 
of a system of large number differential equations, or equivalently, state-space description of high order. So, large number of SISO I-O submodels (easy task) are to be estimated, but they may be integrated in inappropriate system structures.

- It is also known, that mathematical descriptions of MIMO plants in non-parametric forms (frequency function models or impulse response models) allow only P-canonical representation due to the cause-consequence requirements. This limitation, however, could automatically infer to principal errors of third kind. That's why for MIMO systems of mixed or V-canonical structure only parametric model descriptions are fully admissible.

\section{Properties of the MIMO V-Interconnected Plants}

The MIMO plants, possessing V-interconnected structure, are characterized by interactions in reverse direction to that of the causal input-output system relations:

$$
\text { (6) } y_{i}(s)=G_{i i}(s)\left[u_{i}(s)+\sum_{\substack{j=1 \\ j \neq i}}^{n} G_{i j}(s) y_{j}(s)\right], \quad i=1,2, \ldots, n
$$

In vector-matrix notations the model (6) obtains the form

$$
\begin{aligned}
& \mathbf{F}(s) \mathbf{y}(s)=\mathbf{u}(s) \Leftrightarrow \mathbf{y}(s)=\mathbf{W}(s) \mathbf{u}(s), \text { where } \\
& \mathbf{W}(s)=\mathbf{F}^{-1}(s)=\left[\mathbf{I}-\mathbf{G}_{\mathbf{d}}(s) \mathbf{G}_{\mathbf{k}}(s)\right]^{-1} \mathbf{G}_{\mathbf{d}}(s), \\
& (7) \mathbf{G}_{\mathbf{d}}(\mathrm{s})=\operatorname{diag}\left[G_{\mathrm{ii}}(\mathrm{s})\right], i=1, \ldots, n
\end{aligned}
$$

$$
\begin{aligned}
\mathbf{G}_{\mathbf{k}}(s) & =\left[\begin{array}{cccc}
0 & G_{12}(s) & \cdots & G_{1 n}(s) \\
G_{21}(s) & 0 & \cdots & G_{2 n}(s) \\
\cdot & \cdot & \cdots & \cdot \\
G_{n 1}(s) & G_{n 2}(s) & \cdots & 0
\end{array}\right], \\
\text { (8) } \quad \mathbf{F}(s) & =\left[\begin{array}{cccc}
\frac{1}{G_{11}(s)} & -G_{12}(s) & \cdots & -G_{1 n}(s) \\
-G_{21}(s) & \frac{1}{G_{22}(s)} & \cdots & -G_{2 n}(s) \\
\cdot & \cdot & \cdots & \mathrm{i} \\
-G_{n 1}(s) & -G_{n 2}(s) & \cdots & \frac{\mathrm{i}}{G_{n n}(s)}
\end{array}\right] .
\end{aligned}
$$

The formal requirement to P-transformation of a $\mathrm{V}$-model is $\operatorname{det}\left[\mathbf{I}-\mathbf{G}_{\mathbf{d}}(S) \mathbf{G}_{\mathbf{k}}(S)\right] \neq 0$, and $\mathbf{F}$ (s) to be square.

The matrix transfer function $\mathbf{F}_{2 \times 2}(\mathrm{~s})$ of the hydraulic (2x2) V-interconnected plant follows from (8) after substituting $n=2$. Its inverse matrix $\mathbf{W}_{2 \times 2}(\mathrm{~s})=\left[\mathbf{F}_{2 \times 2}(\mathrm{~s})\right]^{-1}$ takes important role to the investigation of $\mathrm{V}$-systems. It is obtaining via (7) after respective transformation in input-output P-form [11]:

$$
\text { (9) } \mathbf{W}_{\mathbf{2} \mathbf{2} 2}^{\mathrm{V}}(s)=\frac{1}{1-\chi_{V}(s)}\left[\mathbf{I}+\mathbf{G}_{\mathbf{d}}(s) \mathbf{G}_{\mathbf{k}}(s)\right] \mathbf{G}_{\mathbf{d}}(s)
$$

where

$$
\text { (10) } \Delta_{V}(s)=1-\chi_{V}(s), \chi_{V}(s)=G_{11}(s) G_{22}(s) G_{12}(s) G_{21}(s)
$$

The common multiplier $\chi_{\mathrm{V}}(\mathrm{s})$ is denoted as the Dynamic Interaction Coefficient of a TITO V-canonical system. He serves as a particular measure for the strength of the cross interactions between channels and shows the main difference between the V-and P-interconnected TITO structures by comparing the respective interaction coefficient $\chi_{\mathrm{P}}(\mathrm{s})$ of TITO P-structures $\chi_{\mathrm{P}}(\mathrm{s})=G_{12}(\mathrm{~s}) G_{21}(\mathrm{~s})\left[G_{11}(\mathrm{~s}) G_{22}(\mathrm{~s})\right]^{-1}$.

If take transformation to $\mathrm{P}$-form with plant matrix $\mathbf{W}^{\mathbf{v}}{ }_{2 \times 2}(\mathrm{~s})$ according to (9), the general TITO V-plant is represented by the equivalent block structure shown on figure 3 , and for the system outputs the following relations are valid:

$$
\text { (11) } y_{i}(s)=\frac{G_{i i}(s)}{1-\chi_{V}(s)} u_{i}(s)+\frac{G_{i i}(s) G_{i j}(s) G_{i j}(s)}{1-\chi_{V}(s)} u_{j}(s), i \neq j=1,2
$$

The asymptotic properties $(t \rightarrow \infty)$ of the process outputs (11), supposing self-control to all dynamic channels, under effect on $u_{1}=u_{2}=1(t) \quad\left(y_{1}(\infty) \equiv y_{2}(\infty)\right)$ or $u_{1}=1(t), u_{2}=0$ $\left(y_{1}(\infty) \neq y_{2}(\infty)\right)$ are as follows:

$$
\begin{aligned}
& y_{i}(\infty)=\left.k_{i i} \frac{1+k_{i j} k_{j j}}{1-\chi_{V}(0)}\right|_{u_{i}=1(t)}, i \neq j=1,2 \\
& y_{1}(\infty)=\left.\frac{k_{11}}{1-\chi_{V}(0)}\right|_{\substack{u_{1}=1(t) \\
u_{2}=0}}, y_{2}(\infty)=\left.\frac{k_{11} k_{21} k_{22}}{1-\chi_{V}(0)}\right|_{\substack{u_{1}=1(t) \\
u_{2}=0}}
\end{aligned}
$$

Here, $\chi_{V}(0)=k_{11} k_{12} k_{21} k_{22}$ is the Static Interaction Coefficient of a TITO V-interconnected plant. By exploiting (12), according to the theoretically possible limit values of the outputs, a special property of the TITO V-interconnected plants is obtained, that takes effect when $\chi^{*}{ }_{V}(0)=1$ for particular values of the plant gains $k^{*}$ ij , always after satisfying the relation:

$$
\text { (13) } \chi_{V}^{*}(0)=k_{11}^{*} k_{12}^{*} k_{21}^{*} k_{22}^{*} \equiv 1
$$

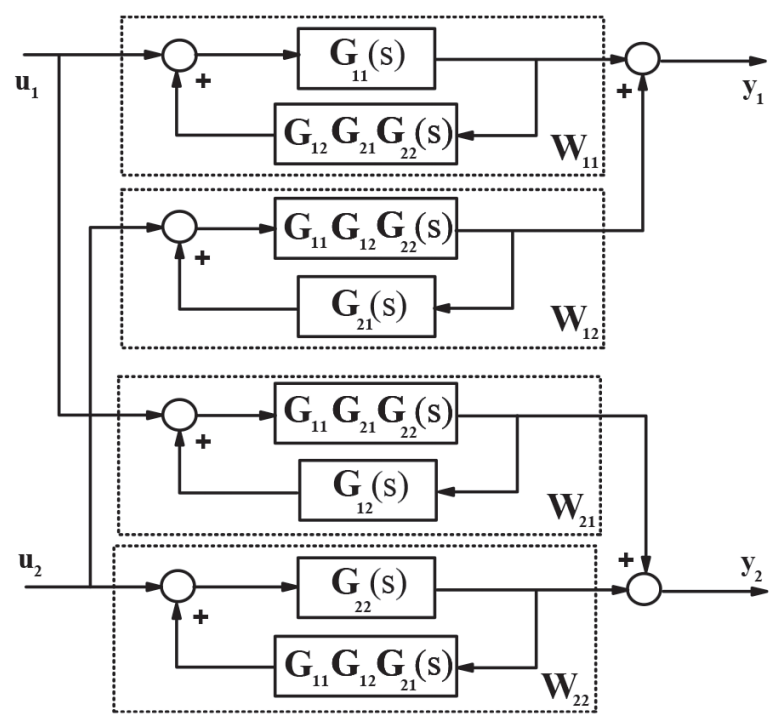

Figure 3. P-matrix W(s). of a (2x2) V-plant 
So, from (13) it follows, that when $\chi_{V}(0) \geq 1$, unconstrained increase of the V-plant outputs will be observed, no matter if all dynamic channels behave partial self-control. At that, if $\chi^{*}{ }_{\mathrm{v}}(0)=1$, then the plant will demonstrate astatic behaviour, and when $\chi_{V}(0)>1$, the plant transients will rise purely unsteady. This theoretical phenomenon shows the existence of an intrinsic particularity to $\mathrm{V}$-interconnected plants for functioning with permanent shifts in their fundamental properties, depending solely by the moment combination between the constructive plant parameters and the load factors of the process. This special parametric property of the V-interconnected plants stipulates for the possibility to odd appearing of structural instability, at that on all system outputs simultaneously.

Qualitative suggestions about available hidden instability in the MIMO systems with reverse interconnections are reported in [6], but they are not regarded to the process internal V-structure, and the MIMO description in V-canonical form is considered as a formal option only, confronting with the alternative and much more convenient P-form. Similar ideas are disposed also in [8] via the notion of "internal loop",

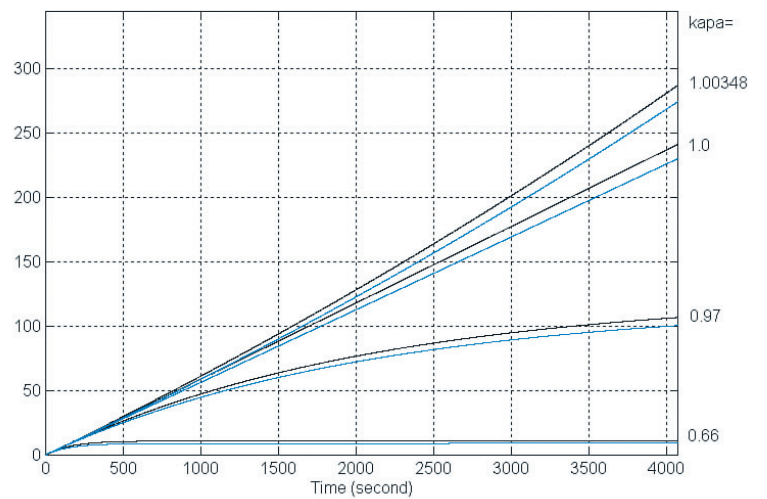

Figure 4. Time responses of a TITO V-interconnected plant for various values of $\chi_{V}(0)$ (Example)

considered able to call forth system instability, if it is realized in the form of positive feedback, but this is not precise. Vice-versa to the cited reference, as early as in Krasovskii [3] it is claimed that the qualitative suggestion for just a single negative feedback available in a MIMO system (i.e. negative V-cross connection from $y_{i}$ to $u_{j}$ )) leads the interconnected V-system to be always stable. Clearly, this condition follows simply from (10), (11), but is not a general rule.

If one applies the definition about characteristic polynomial of a MIMO system in matrix transfer function description to the transformed P-form matrix $\mathbf{W}^{\mathrm{V}}(\mathrm{s})$ of a general (2x2) V-interconnected plant, the polynomial $\mathrm{H}_{2 \times 2}^{\mathrm{V}}(\mathrm{s})$ is obtained, and afterwards - the characteristic equation of the MIMO V-plant:

$$
\begin{aligned}
& H_{2 \times 2}^{V}(s)=\operatorname{Den}\left\{\operatorname{det}\left[\mathbf{W}_{2 \times 2}^{\mathbf{v}}(s)\right]\right\}=\operatorname{Den}\left[1-\chi_{V}(s)\right] \Rightarrow \\
& \operatorname{Den}\left[1-\prod_{i, j=1}^{2} G_{i j}(s)\right]=0
\end{aligned}
$$

When self-control is available to all dynamic channels, then $H_{2 \times 2}^{V}(\mathrm{~s})$ is written in a direct form:

$$
H_{2 x 2}^{V}(s)=1-\prod_{i, j=1}^{2} N_{i j}(s)\left[\prod_{i, j=1}^{2} D_{i j}(s)\right]^{-1}
$$

and afterwards

(15) $\prod_{i, j=1}^{2} D_{i j}(s)-\prod_{i, j=1}^{2} N_{i j}(s)=0$

By comparing (15) with the characteristic equation $H_{2 \times 2}^{P}(s)=\prod_{i, j=1}^{2} D_{i j}(s)=0 \quad$ of the general $(2 \times 2)$ P-interconnected plant, a substantial distinction between $H_{2 \times 2}^{P}(\mathrm{~s})$ and $H^{V}{ }_{2 \times 2}(\mathrm{~s})$ is considered due to the emerging additional polynomials, originating from the $\mathrm{V}$-system matrix numerator. Some more, (15) shows, that theoretical results about MIMO P-structures are no longer valid to V-interconnectivity in general. For a V-interconnected plant, having strict properness by all dynamic channels, $H^{V}{ }_{2 \times 2}(\mathrm{~s})$ has a particular form

$$
\text { (16) } H_{2 x 2}^{V}(s)=\prod_{i, j=1}^{n}\left(T_{i j} s+1\right)-\prod_{i, j=1}^{n} k_{i j}=0,
$$

from which follows the important issue, that the poles of the V-plant are distinguishing considerably by those of a MIMO plant composed by the same transfer functions and P-interconnected internal structure. Moreover, the V-plant poles don't coincide under general conditions with no one of the separate poles (possibly stable) from the component transfer functions $G_{\mathrm{ij}}(\mathrm{s}), i, j=1, \ldots, n$. Furthermore, for $\mathrm{V}$-interconnected MIMO plants, stability can't be guaranteed only by examining the separate poles of the component transfer elements $G_{i j}(\mathrm{~s})$, for the non-trivial inclusion of the numerator polynomials $\prod_{i, j=1}^{2} N_{i j}(s)$, leading the poles of $\prod_{i, j=1}^{2} D_{i j}(s)$ to deviate from the initial (supposing steady) specifications. In

the special case, when $\chi^{*}{ }_{\mathrm{V}}(0)=1$ it follows, that both V-system outputs will demonstrate purely astatic properties and even small variations in plant gains $k_{\mathrm{ij}}$ would produce instability when $\chi_{\mathrm{V}}(0)>1$ to all system outputs $y_{\mathrm{i}}$. That's why the notion of Critical Interaction Gain $\chi_{\mathrm{v}}^{*}(0)$ is introduced [11] to show quantitatively the closeness of the MIMO system to the state of singularity under $\chi^{*}{ }_{\mathrm{V}}(0)=1$. It is evident, that $\chi_{\mathrm{V}}(0)$ depends on the V-plant properties through all channels and formally is specified by the numerator polynomials of $G_{\mathrm{ij}}(s=0)$.

Illustrative example. A strictly proper TITO V-plant with $\chi_{\mathrm{V}}(0)=0.97$ possesses the following characteristic polynomial:

$$
\begin{aligned}
H^{V}(s)= & (6.5 s+1)(5.0 s+1)(7.5 s+1)(8.0 s+1)- \\
& -(1.5)(0.7)(0.44)(2.1)
\end{aligned}
$$

By examining the system transients on figure 4, one could observe system ultra slow responses $\left(\sim 5 \times 10^{3} \mathrm{sec}\right.$ comparing to approx. $150 \mathrm{sec}$ settling time for the independent channels) and large steady-state outputs values 
$\left(y_{1}(\infty)=124.3, y_{2}(\infty)=116.98\right.$, comparing to $k_{\mathrm{ij}} \sim 1.5$ to 2.1$)$. These results follow from the formally stable system poles $(-0.305 ;-0.152 \pm \mathrm{j} 0.1465 ;-0.0011)$, but one deployed too near to the origin $s=0$. This feature follows also by the already defined measure for MIMO V-stability, valued at $\chi_{\mathrm{V}}=0.97$, so being too near the critical level of $\chi_{\mathrm{V}}^{*}=1$. This implies, that if only a single plant model gain "moves" insignificantly, e.g. for $k_{21}=0.44$ to $k_{21}=0.3$, then the value of Static Interaction Coefficient changes to $\chi_{V}(0)=0.66$, and all time responses improve significantly to admissible settling values of $y_{1}(\infty)=7.597, y_{2}(\infty)=3.695$ and $t_{\infty}=400 \mathrm{sec}$ (figure 4 ). For the critical value of $\chi_{\mathrm{V}}=1.0$, obtained by $k_{21}=0.4536$, astatic responses for $y_{i}$ are observing. When $\chi_{v}>1.0$ (e.g. $\chi_{\mathrm{V}}=1.00348$ for $k_{21}=0.456$ ), expected unstable time transients are obtained (the uppermost couple of graphics).

By applying the definition of $R G A$ multivariable index regarding the general TITO V-system, transformed in equivalent P-form, the particular RGA coefficients $\lambda_{\mathrm{ij}}$ are specified, showing distinctions from those of a standard P-structure solely by the expression of $\chi_{\mathrm{r}}(0)$ :

$$
\text { (17) } \lambda_{i i}^{V}=\frac{1}{1-\chi_{V}(0)}, \quad \lambda_{i j}^{V}=-\frac{\chi_{V}(0)}{1-\chi_{V}(0)}, \quad i, j=1,2 \text {. }
$$

Issuing by the fact, that RGA for $\mathrm{V}$-systems are represented by distinct relations (and numeric values, of course) from those to P-plants, different properties regarding the numerous consequences and design solutions on the field of MIMO engineering systems activity and industrial process control are also being waited.

\section{Conclusions and Generalizations}

By the theoretical issues performed and shown illustrative results some basic conclusions could be drawn:

(1) In theoretical aspect, it is fully unjustifiable the inherent structure of the MIMO systems to be neglected for simplifying control problems and identification tasks. It is fully incorrect to adopt ungrounded conceptual "exchange" of a particular canonical structure to another.

(2) The shifts in fundamental properties (stability, self-control) of MIMO V-interconnected plants is proven possible in a set of operating points for arbitrary small variations in system parameters (process gains) under hardly predictable constructive or operating alterations.

(3) It is clear, that the events with changes of the fundamental plant properties in some applied MIMO V-interconnection problems are directly dependent by the particular vessels, processes or installations. This imposes deep theoretical insight on the phenomenon by introducing $\chi \mathrm{V}$ as universal stability measure and diagnostic symptom in the industrial control problems for that class of plants.

\section{References}

1. Isermann, R. Part V. Multivariable Control Systems. Digital Control Systems. Berlin, Springer-Verlag, 1981, 309-347.

2. Johansson, K. H. The Quadruple-Tank Process: A Multivariable Laboratory Process with an Adjustable Zero. - IEEE Trans. on Control System Technology, 8, 2000, No. 3, 456-465.

3. Krasovskii, A. A. - Avtomatika, Telemekhanica, 1957, No. 18, 126-136 (in Russian).

4. Mesarović, M. D. The Control of Multivariable Systems. MIT and Wiley, 1960.

5. Netushil, A. V. Theory of Automatic Control. Moskva, Visshaia Shkola, 1976 (in Russian).

6. Niederlinski, A. A. Heuristic Approach to the Design of Linear Multivariable Interacting Subsystems. - Automatica, 1971, No. 7, 691-701.

7. Ogunnaike, B., W. H. Ray. Chap. 21. Interaction Analysis and Multiple Single-loop Design. Process Dynamics, Modeling and Control. London, Oxford University Press, 1994.

8. Owens, D. H. Multivariable Control for Industrial Applications. Control Eng. Series, 32, John O'Reily (Ed), Chap. 12.

9. Rijnsdorp, J. E. Interaction in Two-variable Control Systems for Distillation Columns - I part. - Automatica, 1,1965, 15-28, 1965.

10. Roffel, B. and B. Betlem. Process Dynamics and Control. Modeling for Control and Prediction. Wiley Padstow, GB, 2006.

11. Tzanev, A. T. Structural Input-output Properties of Low-order MIMO Interconnected Systems. - Int. Journal of Control Engineering and Technology (IJCET), American V-King Scientific Publishing Ltd., 4, 2014, No. 3, 220-242.

\section{Manuscript received on 13.06.2017}

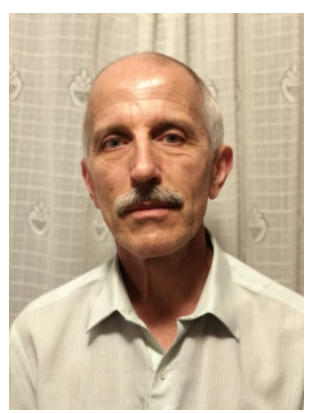

Anjel T. Tzanev, born 1954 in Sofia, Bulgaria. M.Eng in Industrial Process Control (1979), M.Sc. in optimization under uncertainties (1981). Declared PhD degree in Multiloop System Control, 2001. Authorships of scientific reports and journal articles in the areas of multivariable control theory, multiloop and robust control, intelligent control, process modelling and control, Largescale industrial systems (LSS) and Systems of Systems (SoS's). Published monographic didac-tic aids for students in Bulgarian on Process control, original textbook concerning Large-scale Systems control. Invited to create specialized monograph in English (Design of Systems of Systems (SoS) - a Multidisciplinary Approach, 2013) by Lambert Academic Publishing, LAP Inc., Germany. Nowadays appears in charge of General Research coordinator to a Fundamental Scientific Project entitled "Investigation on operational changes in the fundamental properties of MIMO systems, possessing V-type interconnections and developing effective approaches for their control", financed by the National Sci Fund, 2017-2020. Currently engaged as Senior lecturer at Process Control, Control of LSS's and Industrial Automation with the University of Chemical Technology and Metallurgy (UCTM) - Sofia.

Contacts:

Dept. Process Control and Informatics University of Chemical Technology and Metallurgy (UCTM) Sofia

8 Kliment Ohridski Blvd. 1756 Sofia, Bulgaria e-mail:atzanev@uctm.edu 